\title{
Pioderma gangrenoso en mamoplastía de reducción con pedículo inferior*
}

\author{
Drs. WILFREDO CALDERÓN O. ${ }^{1}$, JUAN PABLO CISTERNAS V. ${ }^{2}$, \\ DANIEL CALDERÓN M. ${ }^{2}$, ALEX EULUFI M. ${ }^{1}$, KENNETH GULER G. ${ }^{1}$, \\ LUIS JARAMILLO R. ${ }^{2}$, RODRIGO TAPIA L. ${ }^{2}$, TIRSA SAAVEDRA U. ${ }^{3}$
}

1 Servicio de Cirugía Plástica, Hospital del Salvador.

2 Servicio de Cirugía General, Hospital del Salvador.

3 Dermatología, Clínica Santa María.

Santiago, Chile.

\begin{abstract}
Pyoderma gangrenosum secondary to reduction mammoplasty

Background: Pyoderma gangrenosum (PG) is an uncommon inflammatory and ulcerative disorder of the skin. It may be associated with immune deficiencies. It is usually located in the extremities and its appearance after reduction mammoplasty is exceptional. We report three patients with the disease. A 54 years old female subjected to a bilateral reduction mammoplasty with an inferior pedicle. She developed a pyoderma gangrenosum and was treated with systemic steroids and local application of Dapsone with remission of lesions and healing after one month of evolution. A 23 years old women subjected to the same surgical procedure, which developed a wound dehiscence with ulcerative lesions, was treated with steroids and Azathioprine, reducing the local inflammation but leaving a severe esthetic sequel. A 21 years old woman subjected to the same surgical procedure, develop bilateral wound dehiscence and ulcerative lesions, she was treated with steroids and antimicrobials achieving a secondary healing.
\end{abstract}

Key words: Pyoderma grangrenosum, breast, reduction mammoplasty.

\section{Resumen}

Introducción: El Pioderma Gangrenoso es una enfermedad poco frecuente, de difícil diagnóstico y manejo. Puede estar asociado a otras enfermedades, en donde la alteración de la respuesta inmune es común. Su presentación por lo general corresponde a lesiones ulceradas de la piel ubicadas con frecuencia en las extremidades, siendo su presentación en las mamas excepcional; y mucho más rara su relación post mamoplastía de reducción. Métodos: Se realizó una revisión de pacientes sometidas a mamoplastía de reducción con pedículo inferior, asociadas al desarrollo de pioderma gangrenoso post-quirúrgico, durante un período comprendido

Recibido el 19 de marzo de 2013 y aceptado para publicación el 22 de abril de 2013.

Los autores no refieren conflictos de interés.

Correspondencia: Dr. Wilfredo Calderón O.

Fray Montalva 580, Santiago, Chile.

wilfredocalder@gmail.com 
entre los años 2000 y 2011. Resultados: Se recolectaron 3 pacientes, se describen sus casos clínicos con respecto a esta ubicación, analizando su presentación, evolución y manejo de la enfermedad. Discusión: La presentación del pioderma gangrenoso post-quirúrgico en relación a la mamoplastía de reducción comparte ciertas similitudes, las cuales deben orientar al diagnóstico precoz y de esta forma evitar manejos erróneos que pueden ser deletéreos, secuelantes e incluso potencialmente mortales.

Palabras clave: Pioderma gangrenoso, mamoplastía de reducción, mama.

\section{Introducción}

El Pioderma Gangrenoso (PG) es una rara enfermedad ulcerativa de la piel ${ }^{1}$, que puede causar dolor, desfiguramiento e incluso muerte ${ }^{2}$. Posee una amplia gama de presentación, sin embargo, es frecuente dentro de este espectro el fenómeno de patergía en donde todo intento de aseo quirúrgico o injerto puede desarrollar necrosis y ulceración. Los hallazgos clásicos al examen son úlceras cubiertas con pus, centros necróticos, con bordes violáceos irregulares e indeterminados ${ }^{3}$. Sin embargo, su presentación puede variar desde una úlcera moderada, crónica y localizada, hasta una enfermedad multisistémica asociada principalmente a patologías autoinmunes de rebelde respuesta a tratamiento a las cuales se asocia su patogenia ${ }^{4}$. Esta enfermedad afecta típicamente a extremidades inferiores, pero se puede manifestar en cualquier área anatómica, siendo las mamas una localización inhabitual, por razones aún desconocidas 5 .

La relación de la cirugía mamaria y el PG es un cuadro muy infrecuente, pero está descrita en la literatura, en relación a procedimientos reconstructivos $^{6}$, mamoplastías de aumento ${ }^{7}$ e incluso se han reportado dos series de dos casos cada una ${ }^{8,9}$. La relación del PG y la mamoplastía de reducción es aún más infrecuente, con sólo 8 reportes de casos aislados $^{3,10-16}$.

El objetivo de este trabajo es mostrar una serie de casos, su manejo y realizar una revisión de la literatura.

\section{Método}

Estudio retrospectivo, descriptivo de una serie de casos.

Se realizó una revisión de pacientes sometidas a mamoplastía de reducción con pedículo inferior durante un período comprendido entre los años 2000 y 2011, realizados por un cirujano, y se buscó su asociación con el desarrollo post-quirúrgico de pioderma gangrenoso mamario.

Se describió su edad, presentación clínica, evolución, estudios diagnósticos, manejos iniciales y resultados a corto y mediano plazo.

Finalmente se realizó una revisión de tema.

\section{Resultados}

El primer caso se presentó en una mujer de 54 años portadora de gigantomastia y sometida a mamoplastía de reducción bilateral con pedículo inferior (MRBPI). Inicialmente evolucionó con una herida limpia y seca, pero con aumento de la temperatura local, que luego cambió a bordes eritematosos y abscedados. El estudio reveló alteraciones de laboratorio con leucocitosis y proteína $\mathrm{C}$ reactiva elevada. Fue sometida a aseos quirúrgicos y antibióticos, con mala respuesta, asociándose a úlceras con bordes violáceos-purpúricos, por lo que se planteó el diagnóstico de PG (Figuras 1 y 2). Se realizó estudio histológico inespecífico y se inició tratamiento esteroidal sistémico con prednisona y curaciones con dapsona, tras lo cual se evidenció remisión de las lesiones y cicatrización secundaria completa al mes de evolución (Figuras 3 y 4).

Nuestro segundo caso corresponde a una mujer de 23 años, que padecía de una gigantomastia importante, con distancia de la línea medioclavicular-pezón (LMCP) de $32 \mathrm{~cm}$ bilateral (Figura 5). Se realizó una MRBPI, resecando $1 \mathrm{~kg}$ de tejido por lado. Tras 5 días de evolución favorable, presentó una dehiscencia de sutura de la mama izquierda siendo resuturada; al noveno día presentó nueva dehiscencia, asociándose a lesiones ulcerativas dolorosas, por lo que se planteó el diagnóstico de PG (Figura 6 ), posteriormente se produce dehiscencia total de la mama izquierda (Figura 7), se realizaron estudios de laboratorio que mostraron un patrón inflamatorio, cultivos negativos y una biopsia que describió bordes con infiltrado inflamatorio polimorfonuclear neutrófilo. La dermis superficial con edema, angiectasias e infiltrado inflamatorio linfoplasmocitario y polimorfonuclear neutrófilo, asociado a eosinófilos; siendo estos hallazgos sugerentes de PG. Se inició tratamiento sistémico con prednisona, azatioprina y curaciones de la zona cruenta con Biopiel (Quitosano) (Figura 8), a los 30 días comienza a manifestar una nueva lesión ulcerosa en la mama derecha (Figura 9). Tras aumentar las dosis de azatioprina a 100 $\mathrm{mg}$ /día, comienza reducirse el componente inflamatorio local (Figuras 10 y 11), con buena evolución posterior. En el seguimiento a un año se mantiene en remisión, optándose por un cierre por segunda intención con una severa secuela estética (Figura 12). 


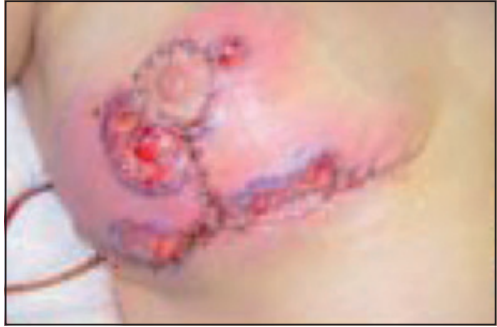

Figura 1. Úlceras múltiples en mama derecha, con bordes eritematosos.

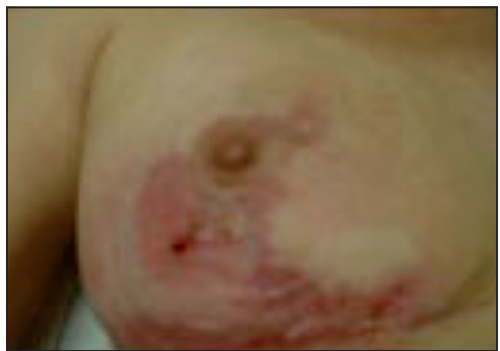

Figura 3. Cicatrización de las lesiones ulcerosas por segunda intención. Mama derecha.

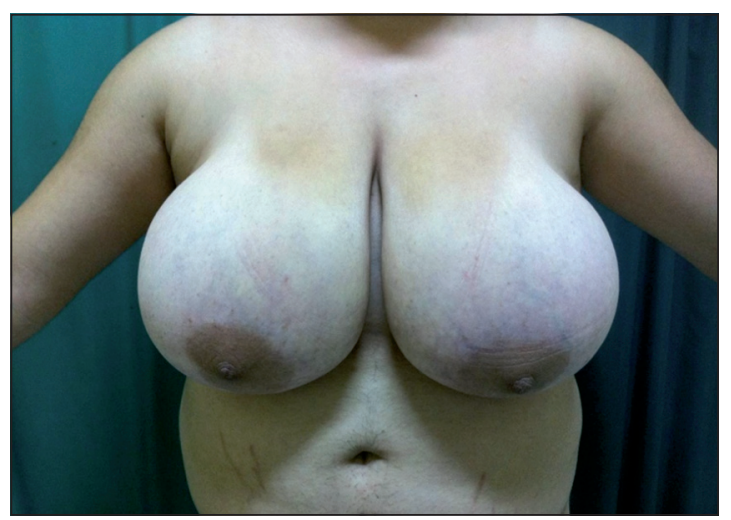

Figura 5. Gran gigantomastia en paciente de 23 años. Distancia LMCP: $32 \mathrm{~cm}$.

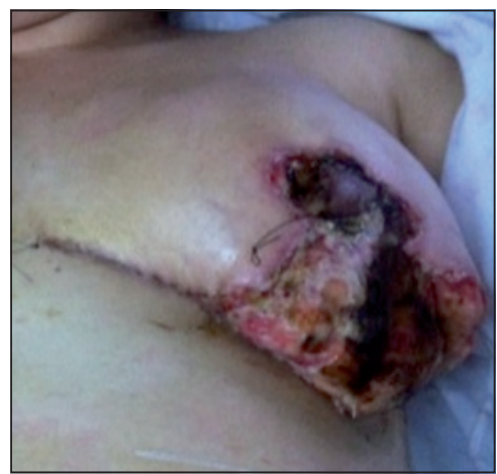

Figura 7. Dehiscencia total de la mama izquierda.

Rev Chil Cir. Vol 65 - № 6, Diciembre 2013; pág. 541-548

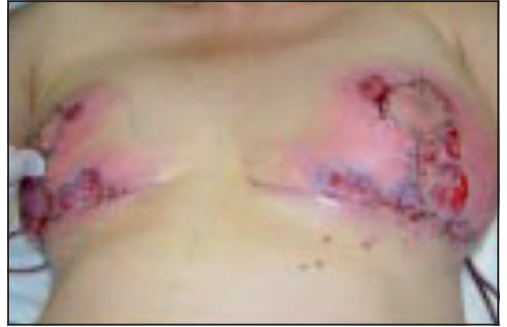

Figura 2. Múltiples lesiones ulcerosas en ambas mamas, con bordes eritematosos.

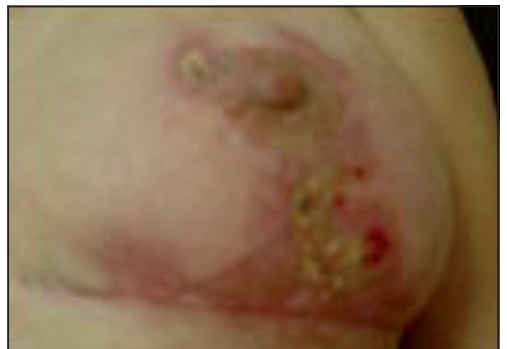

Figura 4. Cicatrización de las lesiones ulcerosas por segunda intención. Mama izquierda.

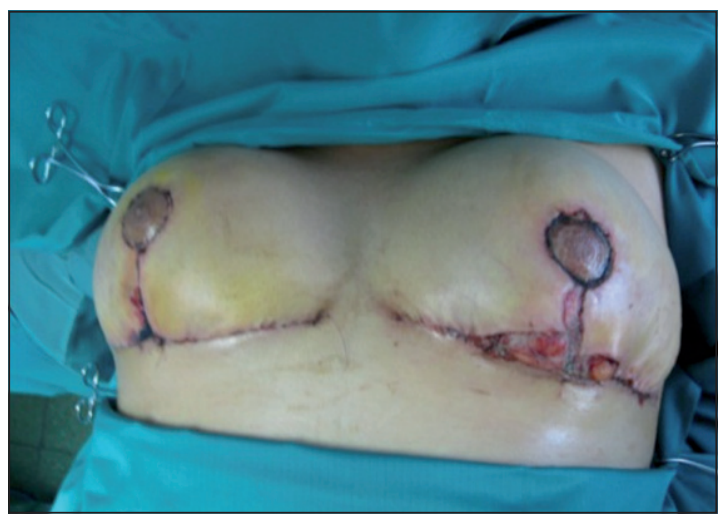

Figura 6. Lesiones ulcerosas, dolorosas, con bordes eritematosos.

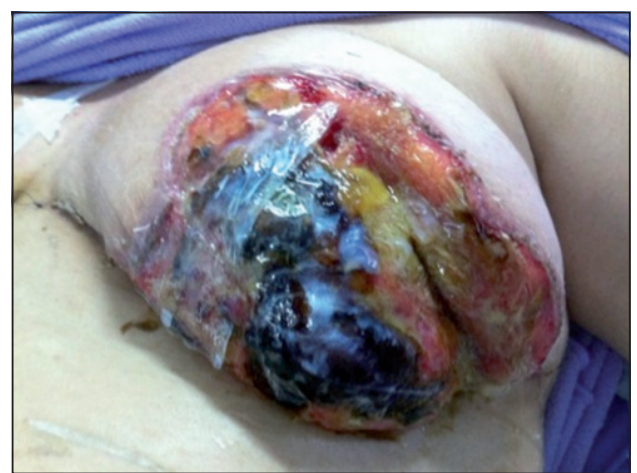

Figura 8. Curación de mama abierta con Biopiel (Quitosano). 


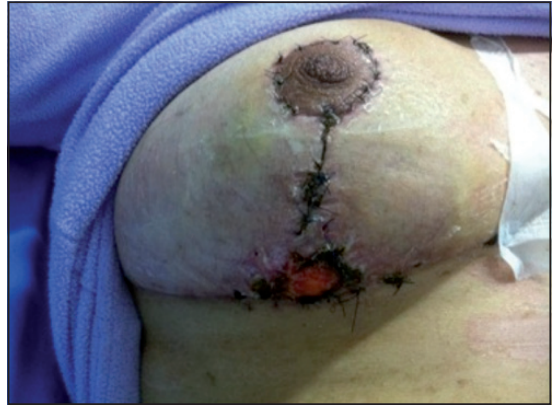

Figura 9. Lesión ulcerosa en convergencia de las líneas de sutura de la mama derecha.

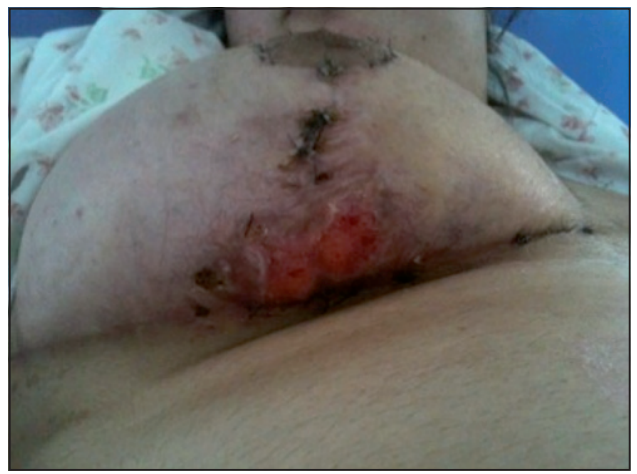

Figura 11. Reducción del componente inflamatorio. Mama derecha.

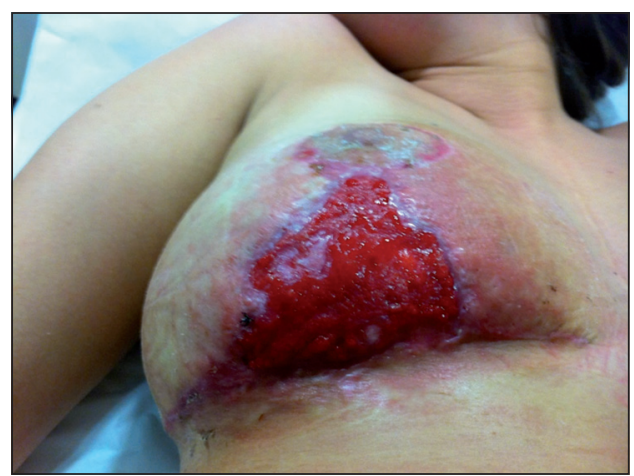

Figura 13. Mama derecha con gran lesión ulcerativa y bordes eritematosos.

El tercer caso presentado es de una mujer de 21 años, con antecedente de gigantomastia que presentaba una distancia de LMCP de $27 \mathrm{~cm}$. Fue sometida a MRBPI resecando $400 \mathrm{~g}$ en cada lado. Con buena evolución postoperatoria durante la primera semana, tras la cual presentó dehiscencia de la línea vertical bilateral, la cual fue resuturada, evolucionando con una segunda recidiva de dehiscencia y ulceración

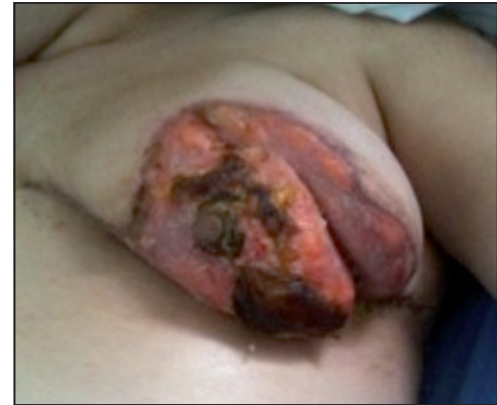

Figura 10. Reducción del componente inflamatorio. Mama izquierda.

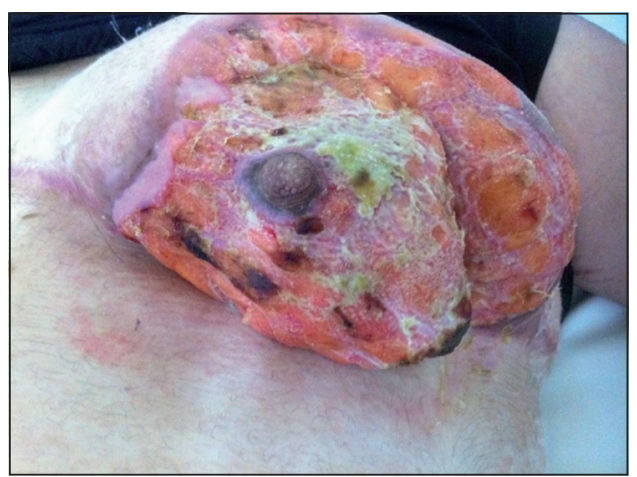

Figura 12. Seguimiento a un año. Mama en remisión con gran secuela estética.

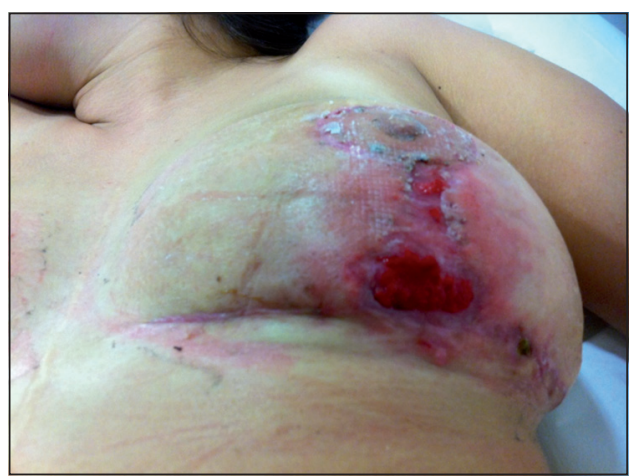

Figura 14. Mama izquierda con lesiones ulcerativas menores, asociados a inflamación local.

bilateral asociada a un componente inflamatorio mayor a derecha (Figuras 13 y 14). Nuevamente en un trabajo multidisciplinario se realizaron estudios de laboratorio e histológicos, que describieron un patrón inflamatorio, con cultivos positivos para Staphilococo aureus e histología con predominancia de infiltrado inflamatorio polimorfonuclear. Tras descartar otras patologías se estableció el diagnósti- 


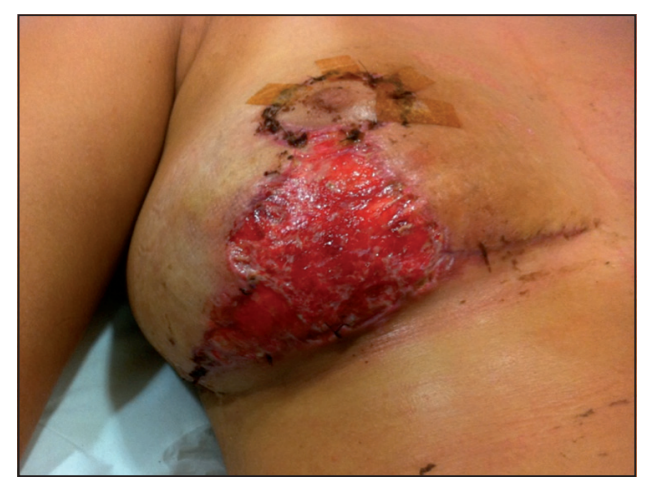

Figura 15. Mama derecha con reducción del componente inflamatorio.

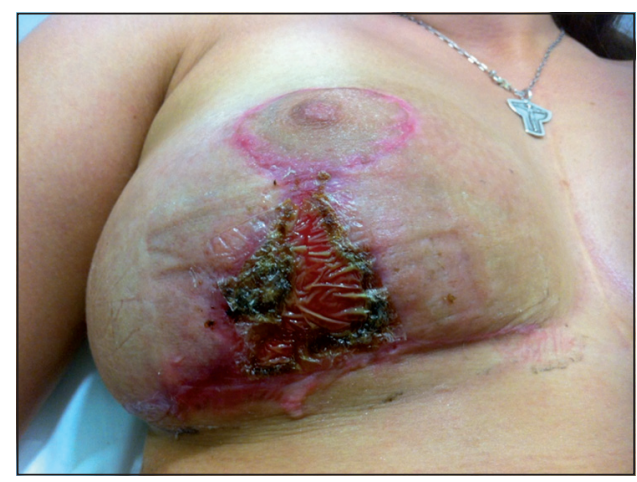

Figura 17. Mama derecha con cicatrización por segunda intención.

co de un PG sobreinfectado y se inició tratamiento precoz basado en prednisona, cotrimoxazol y Biopiel con buena evolución inicial (Figuras 15 y 16), que se mantuvo hasta su cicatrización por segunda intención, evitando así mayores secuelas (Figuras 17 y 18$)$.

\section{Discusión}

La primera descripción del PG la realizó Brocq en 1916 como "phagedenismegeometrique", posteriormente fue caracterizado por Brunsting en $1930^{17}$, presentando cinco pacientes con erupciones cutáneas, de los cuales cuatro presentaban colitis ulcerosa. Lo denominó como "pioderma gangrenoso" debido a que el autor pensó se trataba de una infección estreptocócica que desarrollaba una gangrena cutánea ${ }^{1}$. A pesar de lo errado de la presunción infecciosa del cuadro, la descripción inicial de la patología acertó significativamente en cuanto a la relación patológica del PG con otras patologías. Las más habituales son las enfermedades inflamatorias

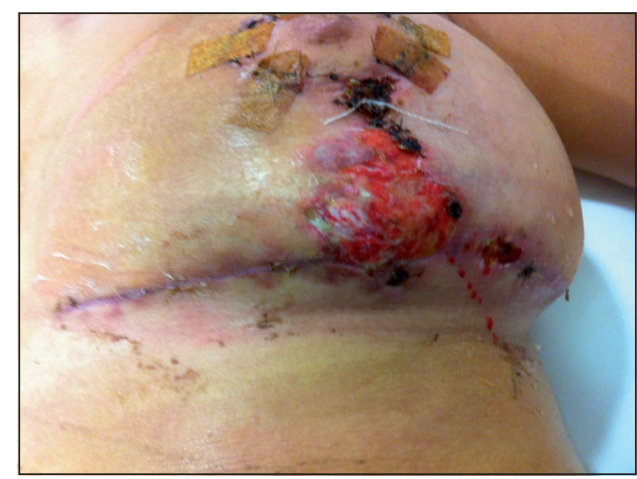

Figura 16. Mama izquierda con reducción del componente inflamatorio.

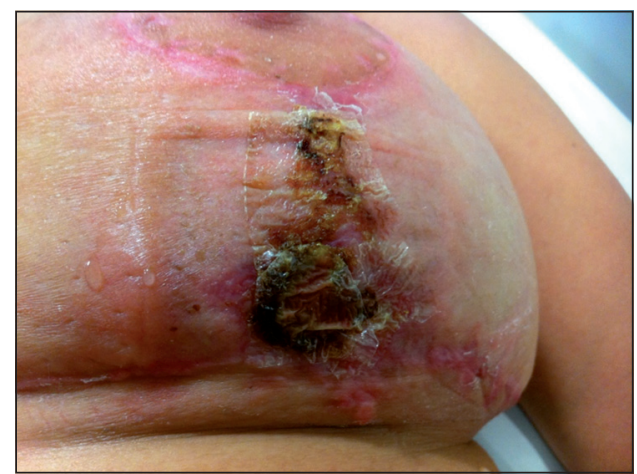

Figura 18. Mama izquierda con cicatrización por segunda intención.

intestinales $(50 \%)$, la artritis reumatoides $(37 \%)$, gammapatía monoclonal $(10 \%)$ y leucemia mieloide aguda o crónica $(7 \%)^{4}$.

Se desconoce la real incidencia del PG. No hay datos para suponer que sea más frecuente en hombres o en mujeres, aunque hay estudios que muestran mayor ocurrencia en mujeres jóvenes especialmente el grupo etario entre los 30 y 50 años, sin embargo, también puede presentarse en el grupo pediátrico ${ }^{4}$. Su escasa frecuencia hace que centros de referencia no informen más de dos o tres casos nuevos al año ${ }^{4}$.

Su descripción clásica es la presencia de una o más lesiones ulceradas cutáneas, dolorosas, de aspecto infeccioso, secreción purulenta, sin mal olor, generalmente con estudio bacteriológico negativo de no coexistir sobreinfección ${ }^{4}$, con bordes irregu$\operatorname{lares}^{1}$ y con una desmesurada respuesta al trauma local (curaciones, aseos quirúrgicos) denominado patergia ${ }^{4}$.

La úlcera a menudo comienza como una pequeña pápula o colección de pápulas, que se rompen para 
formar pequeñas úlceras. Estas tienden a coalescer y el área central presenta luego necrosis y forma una sola úlcera ${ }^{1}$.

Esta patología generalmente se clasifica en 4 tipos: ulcerativa (clásica), bulosa, pustular, y vegetativa $^{2}$. De estas variantes clínicas, la forma ulcerativa es por lejos la más prevalente ${ }^{18}$.

No existe ningún marcador diagnóstico específi$\mathrm{co}^{4}$. El diagnóstico es básicamente por presunción y descarte de otras hipótesis etiológicas, incluso a veces asociado a una prueba terapéutica basada en el uso de corticoides intralesionales. Debido a la agresividad del cuadro, este puede ser fácilmente confundido con una fascitis necrotizante del tipo Estreptococo ß-hemolítica o infección por anaerobios como el Clostridium perfingens ${ }^{4}$. Otra pista que orienta al diagnóstico es el severo y desproporcionado dolor en relación a las lesiones ${ }^{3}$.

El laboratorio muestra un patrón infeccioso, donde priman la leucocitosis y las formas juveniles, con proteína $\mathrm{C}$ reactiva (PCR) elevada y cultivos de herida o hemocultivos negativos ${ }^{4}$.

El estudio anátomo-patológico informa generalmente tejido inflamatorio inespecífico, aunque algunos autores describen vasculitis cutánea, principalmente necrotizante ${ }^{4}$. La histopatología de PG depende del momento de la toma de biopsia y del sitio. Las biopsias tomadas precozmente en la enfermedad y desde los bordes eritematosos progresivos, tienden a mostrar un infiltrado de células inflamatorias crónicas confinadas a la dermis. Ellas a menudo tienen características sugerentes de vasculitis en los bordes de las úlceras, con un infiltrado perivascular linfocítico y necrosis fibrinoide de la pared dérmica de los vasos ${ }^{3}$. Ocasionalmente, la extravasación de los glóbulos rojos y áreas de trombosis también son vistas. Las biopsias tomadas tardíamente en el curso de ulceración, usualmente muestra un infiltrado celular polimorfonuclear con características de ulceración, infarto y formación de abscesos ${ }^{4}$.

Ya que no hay exámenes de laboratorio patognomónicos o hallazgos histológicos específicos, el diagnóstico de PG es principalmente basado en características clínicas y el curso de las lesiones, desórdenes sistémicos asociados, exclusión de otras condiciones y respuesta al tratamiento. El examen histológico es especialmente útil para distinguir el PG de otras causas de ulceración ${ }^{2,5}$. Sin embargo, en presencia de enfermedad ulcerosa con infiltración dérmica neutrofílica (como el caso de úlceras infectadas agudas) esta distinción puede ser desafiante y a veces imposible 5 .

El PG presenta un desafío diagnóstico durante la fase post-operatoria, debido a su similitud con las infecciones de heridas postoperatorias y su rara apariencia $^{\mathrm{d}}$.
El tratamiento, primero, debe enfocarse en el manejo de cualquier enfermedad subyacente que esté presente. Muchas veces estas patologías se relacionan con una respuesta inmune inadecuada la cual puede desencadenar PG. Su resolución puede conducir también a la cura de PG, aunque algunas veces esta se comporta en forma independiente a la enfermedad asociada ${ }^{19}$.

En el tratamiento sistémico, uno de los esteroides más usados es la prednisona en dosis de 1-2 mg/ $\mathrm{Kg} /$ dia, debido a su rápida respuesta (2-3 días) ${ }^{1}$. De igual forma se ha utilizado metilprednisolona en pulsos de $1 \mathrm{~g}$ al día con respuesta entre 3-5 días. Sin embargo, algunas úlceras pueden requerir meses o años para resolverse completamente y/o son refractarias al tratamiento esteroidal ${ }^{2}$. Por tanto, otros inmunomoduladores han sido usados. De estos el más usado es la ciclosporina, que puede ayudar como agente adyuvante de los corticoides en dosis de $5-10 \mathrm{mg} / \mathrm{Kg} / \mathrm{día}^{4}$. Sin embargo, la ciclosporina tiene sus propios efectos colaterales, incluyendo toxicidad renal con el uso prolongado ${ }^{2}$. El metotrexato, azatioprina, y talidomida también han sido usados, pero son generalmente más efectivos como terapias adyuvantes más que tratamientos de primera línea ${ }^{1}$. La Dapsona en combinación con prednisona o sola como terapia de mantención es tambien usada. El uso de tacrolimus en forma local y sistémica también ha mostrado una buena respuesta ${ }^{1,2,4}$.

Con excepción de las variantes vegetativas, los pacientes con PG casi universalmente experimentan dolor, por tanto, se debe implementar analgesia. La fuente de dolor es multifactorial, pero gran parte se atribuye a procesos inflamatorios de PG en la dermis y la úlcera profunda resultante ${ }^{15}$. La manipulación repetida de la herida, inherente con los cambios regulares de curaciones, es fuente de discomfort continuo para el paciente ${ }^{3}$. Tan importante como cuantificar el tamaño y progresión de la lesión, es documentar el nivel del dolor como marcador de eficacia del tratamiento. Cuando una inflamación lesional es adecuadamente tratada y el cuidado de la herida es apropiadamente agresivo, el dolor debiera ceder ${ }^{4}$.

En el tratamiento local, las curaciones que mantienen un ambiente humectante parecieran ser superior a las demás; ya que proveen mejor control del dolor, inducen producción de colágeno, facilitan el debridamiento autolítico y promueven la angiogénesis ${ }^{2}$. Además, crean una barrera a la infección, que es particularmente relevante en PG, ya que su tratamiento sistémico puede impedir una respuesta fisiológica inmune a la invasión bacteriana. Se deben evitar los aseos quirúrgicos y debridamientos de la herida por el fenómeno de patergia asociado a PG. En cada visita, la medición objetiva de la herida in- 
cluye profundidad, largo y ancho; observando en la evolución si existe elevación de bordes y expansión de lesiones.

El uso de cámaras hiperbáricas ha sido usado en vez de inmunosupresores, con algunos resultados favorables ${ }^{20}$, sin embargo, no es fácilmente disponible ${ }^{3}$.

El manejo quirúrgico de $\mathrm{PG}$ es controversial. El debridamiento simple y uso de injerto sobre las úlceras ha sido usado, pero con resultados que pueden ser desalentadores e incluso puede exacerbar la enfermedadi; por otro lado, el manejo conservador lleva a cierre por segunda intención con importantes secuelas cicatriciales ${ }^{10-12}$.

Si se prefiere optar por la alternativa quirúrgica, existen tres recomendaciones: primero, siempre usar terapia inmunosupresora preoperatoria y mantenerla por al menos 6 meses postoperatoria ${ }^{21}$; segundo, evitar usar suturas epidérmicas, preferir cintas adhesivas, de esta forma se podría prevenir el desarrollo de PG en el sitio de sutura' ${ }^{22}$; tercero, si está considerando el uso de injertos, preferir aloinjertos con cobertura esteroidal para minimizar el dolor y favorecer la cicatrizacion ${ }^{3}$.

\section{Conclusión}

Es interesante observar el grado de similitud de las secuencias clínicas presentadas tanto en este reporte como en los demás revisados, que incluso han llevado a algunos autores a distinguir a la forma de presentación post-quirúrgica del PG como una entidad clínica en sí. Todas nuestras pacientes, tras un postoperatorio precoz normal, que osciló entre 3 y siete días, presentan un cuadro inflamatorio progresivo asociado a lesiones ulcerosas que responden negativamente al tratamiento antibiótico y debridativo. El manejo multidisciplinario, en conjunto con dermatología y reumatología, entre otras especialidades, resultó fundamental en estos casos para descartar otras patologías, establecer el diagnóstico y poder manejar a estas pacientes de una manera efectiva y segura.

En resumen, el reconocimiento y tratamiento precoz es importante para prevenir serias morbilidades físicas y psicológicas.

\section{Referencias}

1. Brooklyn T, Dunnill G, Probert C. Diagnosis and treatment of Pyoderma gangrenosum. BMJ. 2006; 333:181-4.

2. Miller J, Yentzer BA, Clark A, Jorizzo JL, Feldman SR. Pyoderma gangrenosum: a review and update on new therapies. J Am Acad Dermatol. 2010;62:646-54.

3. Horner B, El-Muttardi N, Mercer D. Pyoderma gangrenosum complicating bilateral breast reduction. Br J Plast Surg. 2004;57:679-81.

4. Eulufí MA, Calderón OW, Piñeros BJL, Silva CM, Cuadra CA, Léniz MP y cols. Pioderma gangrenoso: reporte de 3 casos en cirugìa plástica. Rev Med Chile 2006;134:339-44.

5. Mansur AT, Balaban D, Göktay F, Takmaz S. Pyoderma gangrenosum on the breast: a case presentation and review of the published work. J Dermatol. 2010;37:10710.

6. MacKenzie D, Moiemen N, Frame JD. Pyoderma gangrenosum following breast reconstruction. Br J Plast Surg. 2000;53:441-3.

7. Sotillo-Gago I, Muñoz-Pérez MA, Camacho-Martínez F. Pyoderma gangrenosum after augmentation mammoplasty. Acta Derm Venereol. 1999;79:486.

8. Davis MD, Alexander JL, Prawer SE. Pyoderma gangrenosum of the breasts precipitated by breast surgery. J Am Acad Dermatol. 2006;55:317-20.

9. Haenen C, ten Berge RL, Posch NA, Braam MJ. Pyoderma gangrenosum following breast surgery. Ned Tijdschr Geneeskd. 2012;156:A4984.

10. Berry MG, Tavakkolizadeh A, Sommerlad BC. Necrotizing ulceration after breast reduction. J R Soc Med. 2003;96:186-7.

11. Clugston PA, Thompson RP, Schlappner OL. Pyoderma gangrenosum after reduction mammoplasty. Can J Surg. 1991;34:157-61.

12. Gudi VS, Julian C, Bowers PW. Pyoderma gangrenosum complicating bilateral mammaplasty. Br J Plast Surg. 2000;53:440-1.

13. Gulyas K, Kimble FW. Atypical Pyoderma gangrenosum after breast reduction. Aesthetic Plast Surg. 2003;27:328-31.

14. Lifchez SD, Larson DL. Pyoderma gangrenosum after reduction mammaplasty in an otherwise healthy patient. Ann Plast Surg. 2002;49:410-3.

15. Grau Salvat C, Miquel FJ, Pont V, Aliaga A. Pyoderma gangrenosum: unusual complication following mammoplasty reduction. Int J Dermatol. 1998;37:794-6.

16. Grillo MA, Cavalheiro TT, da Silva Mulazani M, Rocha JL, Semchechen D, da Cunha CA. Postsurgical Pyoderma gangrenosum complicating reduction mammaplasty. Aesthetic Plast Surg. 2012;36:1347-52.

17. Brunsting LA, Goeckenman WH, O'Leary PA. Pyoderma gangrenosum: clinical and experimental observations in five cases occurrig in adults. Arch Dermatol. 1930;22:655.

18. Poucke SV, Jorens PG, Peeters R, Jacobs W, de Beeck BO, Lambert J, et al. Pyoderma gangrenosum: a challenging complication of bilateral mastopexy. Int Wound J. 2004;1:207-13.

19. Dabade TS, Davis MD. Diagnosis and treatment of the neutrophilic dermatoses (Pyoderma gangrenosum, 
W. CALDERÓN O. y cols.

Sweet's syndrome). Dermatol Ther. 2011;24:273-84.

20. Davis JC, Landeen JM, Levine RA. Pyoderma gangrenosum: skin grafting after preparation with hyperbaric oxygen. Plast Reconstr Surg. 1987;79:200-7.

21. Kaddoura IL, Amm C. A rationale for adjuvant surgical intervention in Pyoderma gangrenosum. Ann Plast Surg. 2001;46:23-8.

22. Long CC, Jessop J, Young M, Holt PJ. Minimizing the risk of post-operative Pyoderma gangrenosum. $\mathrm{Br} \mathrm{J}$ Dermatol. 1992;127:45-8. 